\title{
Session 4: Advances in technology
}

\author{
Wednesday 12th November 2008. Moderator: Mark Glassy
}

\section{[16.10-16.40]}

\section{What is the potential for antibody fragments?}

David P Humphreys

UCB-Celltech, Slough, UK

Proteolytic and engineered Fab' fragments have been found to be invaluable as research tools in the laboratory and clinic for decades. This potential has now been extended to their successful therapeutic use, with ReoPro $^{\mathrm{TM}}$ (Eli Lilly), Lucentis ${ }^{\mathrm{TM}}$ (Genentech) and $\mathrm{Cimzia}^{\mathrm{TM}}$ (UCB) approved for sale and Mycograb ${ }^{\mathrm{TM}}$ (Novartis) successfully completing phase III clinical trials. In addition to Fab', a whole spectrum of engineered antibody fragment derivatives and mimetics are being investigated as therapeutics, diagnostics and reagents due to their alternative biophysical and expression properties.

We will compare and contrast the various formats of antibody fragments with whole antibodies in terms of their current and potential end uses. This will be illustrated with special reference to UCB-Celltech's experience with production and clinical use of PEGylated $\mathrm{Fab}$ and $\mathrm{F}(\mathrm{ab})_{2}$.

\section{[16.40-17.10]}

Capturing the natural humoral immune response by Symplex

John Haurum

Symphogen AS, Lyngy, Denmark

The Symplex technology captures the natural humoral immune response and enables the efficient discovery of target-specific fully human recombinant antibodies drug lead candidates.

The utility of Symplex has been verified by isolating large and diverse sets of unique, fully human antibodies from naturally immune or vaccinated donors with antibody responses against viruses and bacteria, e.g. vaccinia virus, respiratory syncytial virus (RSV), and tetanus toxoid.
The isolated antibodies are high affinity, demonstrate broad antigen and epitope recognition and have potent biological activity.

Molecular analysis of representative human $\mathrm{Ab}$ repertoires will be presented, as well as data demonstrating synergy of antibody mixtures against categories of antibody targets.

We present here a unique approach for the discovery of antibody-based therapeutics, which holds promise for a more efficacious therapeutic approach against in infectious diseases.

\section{[17.10-17.30]}

Anti-thrombopoietin receptor agonist antibodies improved by domain-subclass conversion Masayuki Kai, Kazuhiro Motoki, Hideaki Yoshida, Hiroshi Miyazaki and Shiro Kataoka Discovery Research Laboratories, Kirin Pharma Co. Ltd., Japan

Thrombopoietin (TPO) is a hematopoietic cytokine that regulates megakaryopoiesis and platelet production. Two types of recombinant TPO - full-length recombinant human TPO and pegylated recombinant human megakaryocyte growth and development factor (Peg-rhMGDF), a molecule consisting of the receptor-binding portion of human TPO conjugated to a polyethylene glycol moiety — have been clinically tested in a variety of thrombocytopenic disorders. However, the development was thwarted by the immunogenicity of Peg-rhMGDF. The problem induced attempts at clinical development of TPO-mimetic molecules. Here we report the generation of novel anti-c-Mpl agonist antibodies whose activity was enhanced by switching the subclass of their constant region. Our results suggest the importance of the hinge region in modulating agonist activity. The antibodies showed TPO-like activity in vitro and in vivo, as well as a good pharmacokinetic profile based on the whole-IgG structure, and thus may provide a new medical option for the treatment of thrombocytopenia. 


\section{References}

[1] M. Kai et al., Switching constant domains enhances agonist activities of antibodies to a thrombopoietin receptor, Nat Biotechnol 26(2) (Feb. 2008) 209-211.

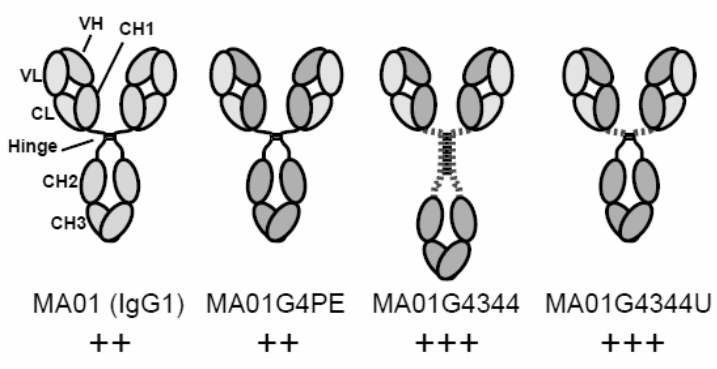

[17.30-17.50]

\section{Plant farming of antifungal recombinant} antibodies

Capodicasa C. ${ }^{\mathrm{a}}$, Torosantucci A. ${ }^{\mathrm{b}}$, Chiani P. ${ }^{\mathrm{b}}$, Bromuro C. ${ }^{\mathrm{b}}$, Cassone A. ${ }^{\mathrm{b}}$ and Benvenuto E. ${ }^{\mathrm{a}}$ ${ }^{a}$ ENEA, Dipartimento BIOTEC, Sezione Genetica e Genomica Vegetale, C.R. Casaccia, 00123 Roma, Italy ${ }^{\mathrm{b}}$ Dipartimento di malattie infettive, parassitiche e immunomediate, Istituto Superiore di Sanità, 00186 Rome, Italy

Infections by opportunistic fungal agents, in particular candidiasis and aspergillosis, cause a high morbility and mortality in immunodepressed and hospitalized patients. In a recent work [1] a specific anti-ßglucan monoclonal antibody was generated in vaccinated mice. This $\mathrm{mAb}$, named $2 \mathrm{G} 8$, bound to the cell wall polysaccharide of Candida albicans hyphae inhibiting their growth in vitro and exerting in vivo a protective effect in models of systemic and vaginal infections.

The variable regions, $\mathrm{VH}$ and $\mathrm{VL}$, genes of this murine antibody have been cloned from the cDNA of mAb2G8 expressing hybridoma and engineered to obtain novel anti-ß-glucan antibodies in different formats:

i) a simple $\mathrm{scFv}$ antibody (fusing the variable domains through a linker peptide);

ii) a scFv-Fc minibody (fusing the $\mathrm{scFv} 2 \mathrm{G} 8$ to a human IgG1 hinge, $\mathrm{CH} 2, \mathrm{CH} 3$ domains in a single protein able to dimerize in a complete antibody);

iii) a human chimeric antibody (fusing murine $\mathrm{VH}$ and VL genes to $\mathrm{CH}$ and CL genes of a human $\mathrm{IgG} 1$ ).

In order to exploit the potential of plants as ideal cost-effective expression system, the two full-length antibodies (human chimeric and $\mathrm{scFv}-\mathrm{Fc}$ ) have been transiently expressed in Nicotiana benthamiana plants by the vacuum-agroinfiltration technique. Western blot analysis of agroinfiltrated plant extracts revealed high expression levels of both chimeric light and heavy chains and of scFv-Fc. The full antibody and the scFvFc were purified from leaves by protein A affinity chromatography with very high yields (40 and $50 \mathrm{mg} / \mathrm{kg}$ of plant tissue, respectively). The correct assembly of both purified antibodies was evaluated by gel filtration and non reducing SDS-PAGE analysis. The binding specificity of purified human chimeric IgG and scFv-Fc was assayed by ELISA. Moreover, both antibodies bind $C$. albicans and A. fumigatus hyphae in immunofluorescence and ELISA assays and inhibit growth of $\mathrm{C}$. albicans in vitro. In addition, the $\mathrm{scFv}$ format expressed only in E. coli periplasm and purified by IMAC, retained the binding specificity of $2 \mathrm{G} 8 \mathrm{mAb}$ in both immunofluorescence and ELISA assays.

In conclusion, we have generated a panel of recombinant antibodies, derived from a murine protective antiß-glucan monoclonal antibody. All recombinant antibody formats retain binding specificity of the original $\mathrm{mAb}$ therefore could represent valuable tools for both diagnostics and immunoprophylaxis/therapy of human fungal diseases. The high yield obtained in plants confirm the notion that plants represent ultimate bioreactors for the expression of fully functional monoclonal antibodies.

\section{References}

[1] Torosantucci et al., A novel glycoconjugate vaccine against fungal pathogens, J Exp Med 202 (2005), 597-606.

\section{[17.50-18.10]}

The humanization of pharmaceutical glycoproteins using targeted expression or trapping in the plant secretory pathway

V. Gomord and L. Faye

CNRS-University of Rouen, France

While N-glycan synthesis in the endoplasmic reticulum (ER) is conserved in plants and mammals, Nglycan maturation in the Golgi apparatus is plantspecific and results in different oligosaccharide structures attached to glycoproteins in plants and mammals. In the prospect of using plants as alternative hosts to mammalian cell lines for production of therapeutic glycoproteins, significant progresses have been 
made towards humanization of protein $\mathrm{N}$-glycosylation in plants.

To date, most efforts to humanize the N-glycosylation pathway in plants have focused on the knock out of genes that code for plant-specific glycosyltransferases together with the knock in of genes catalyzing the synthesis, transport and addition of human sugars.

We are currently taking advantage of a large panel of signals available for targeting of proteins in the membranes of the plant secretory pathway to engineer $\mathrm{N}$-glycosylation of plant-made pharmaceuticals. 